\title{
Vorwort zur zehnten Auflage.
}

Die umgearbeitete und vermehrte neunte Auflage der „Natürlichen Schöpfungsgeschichte", welche vor vier Jahren erschien, ist in der vorliegenden zehnten Auflage nur wenig verändert worden. Abgesehen von zahlreichen Verbesserungen im einzelnen, ist Inhalt und Umfang des Textes derselbe geblieben. Insbesondere habe ich keine Veranlassung gefunden, mein neues $\mathrm{Ph}$ y log en et is ches Sy tem der organischen Formen, wie es in der ,Allgemeinen Stammesgeschichte" des zweiten Teiles übersichtlich dargestellt ist, wesentlich zu verändern. Der Leser, welcher sich für die eingehende wissenschaftliche Begründung desselben interessiert, findet sie in den drei Bänden meiner,, $\mathrm{S}$ y s t e $\mathrm{m}$ a t is chen Phy logen ie" (1894-96).

Inzwischen sind die großen allgemeinen Fragen der menschlichen Naturerkenntnis, für welche mit der Begründung der Entwicklungslehre vor vierzig Jahren eine neue Epoche begonnen hat, in den letzten vier Jahren Gegenstand der lebhaftesten geistigen Kämpfe geworden. Während einerseits das erstaunliche Wachstum unserer empirischen Kenntnisse von den Naturerscheinungen zu einer weitgehenden Arbeitsteilung und Zersplitterung in speziellen Beobachtungen und Experimenten führte, wuchs andererseits in gleichem Maße das Bedürfnis, in diesem grenzenlosen Chaos der Spezialforschung allgemeine Gesichtspunkte für die vernünftige Beurteilung zu finden, kausale Gesetze für die Erklärung des Zusammenhanges anfzustellen. Gegenüber jener Beschränkung der ,,beschreibenden" Naturwissenschaft machte sich wieder das Bedürfnis des denkenden Menschengeistes nach einer vernünftigen Weltanschauung, nach einer naturgemäßen Philosophie geltend.

So erklärt sich die erfreuliche Erscheinung, daß das Ende des abgelaufenen neunzehnten Jahrhunderts zu einem neuen Aufschwunge der vielverdächtigten $\mathrm{N}$ a t u r p hil o s o p h i e geführt hat - nicht zurück zu jenen abenteuerlichen und mystischen Spekulationen, welche im Anfang des neunzehnten Jahrhunderts das Nachdenken über die beobachteten Naturerscheinungen überhaupt in Mißkredit gebracht hatten, sondern zu nüchternen und besonnenen Betrachtungen über den ursächlichen Zusammenhang der zahllosen Einzelheiten, zu einer gesunden Entwicklungslehre auf breitester empirischer Basis. In diesem Sinne hatte ich schon vor 36 Jahren die Grundlinien einer naturgemäßen $\mathbf{M}$ o $\mathrm{n}$ i s t $\mathrm{i}$ $\mathrm{schen} \mathrm{Ph}$ il o s o phie im ersten Buche meiner "Generellen Morphologie der Organismen" festzulegen versucht. Indessen hatte dieser erste Versuch nur sehr geringen Erfolg. Um so größer war die Wirkung meines Buches über,,die Weltr ät sel", in welchem ich vor drei Jahren, die weitere Ausführung, Begründung und Ergänzung" meines ",Monismus" gab. ${ }^{85}$ Von diesen 
„,Gemeinverständlichen Studien über Monistische Philosophie“ erschienen innerhalb zwei Jahren sechs Auflagen (in dreizehntausend Exemplaren); und auch das Ausland bekundete seine Teilnahme an denselben durch zahlreiche Übersetzungen. Die Leser der Natürlichen Schöpfungsgeschichte, welche in deren erstem und letztem Vortrage die allgemeine Bedeutung der modernen Entwicklungslehre für die höchsten Fragen der Philosophie nur kurz angedeutet finden, erhalten in dem Buche über die Welträtsel diejenige ausführliche Begründung derselben, welche ich nach einer gewissenhaften, seit vierzig Jahren darauf gerichteten Lebensarbeit allein zu geben vermag. In dem ersten Teile dieses Buches ist der $\mathrm{M}$ e $\mathrm{n} \mathrm{s} \mathrm{C} \mathrm{h}$ behandelt, im zweiten die $\mathrm{S}$ e e l e, im dritten die $\mathrm{W}$ e I t , im vierten der G ot t.

Wie lebhaft das Interesse der gebildeten Kreise an diesen höchsten Fragen des Menschengeistes jetzt wieder ist, und welche hohe Bedeutung demgemä $\beta$ unsere ,monistische Entwicklungslehre", als der natürliche Schlüssel zur Lösung derselben, erlangt hat, zeigt am deutlichsten der fortdauernde , Kampf um die Welträtsel“". (Vgl. S. 639, Nr. 86.)

Obgleich in der neunten Auflage der ,,Natürlichen Schöpfungsgeschichte" die erläuternden Illustrationen beträchtlich vermehrt und die Gesamtzahl der Tafeln von 20 auf 30 gesteigert war, wurde doch vielfach der Wunsch nach weiterer Vermehrung derselben ausgesprochen. Diesem Wunsche bin ich durch Herausgabe eines neuen Werkes nachgekommen, welches seit 1899 unter dem Titel:, K u n s f or mender N a t u r " im Verlage des Bibliographischen Institutes in Leipzig erscheint. In den zehn Heften desselben habe ich auf 100 Tafeln diejenigen Formen des Tier- und Pflanzenreiches zusammengestellt, welche durch besondere Schönheit und eigenartige Gestaltung sich auszeichnen. Allerdings ist in diesem Werke der ä $\mathrm{s} t \mathrm{~h}$ e $\mathrm{t}$ i s c h e Zweck überwiegend, der Wunsch, in weiteren Kreisen, namentlich der Künstler und des Kunstgewerbes, die Fülle von schönen und interessanten Formen bekannt zu machen, die besonders in den niederen Kreisen des kleinsten Lebens verborgen liegt. Aber daneben habe ich stets den wis sen s c haft li c he n Zweck im Auge behalten und aus der unendlichen Fülle der organischen Gestalten diejenigen ausgewählt, welche zugleich die Kenntnis und das Verständnis der interessantesten Lebensbildungen fördern. Somit bilden die ,"Kunstformen der Natur" (deren letzte Hefte noch in diesem Jahre erscheinen sollen) zugleich einen illustrierenden F or m e n a t l a s zur ,Natürlichen Schöpfungsgeschichte“.

J e n a, am 2. April 1902.

Ernst Haeckel. 